NASZA DERMATOLOGIA Online OUR DERMATOLOGY Online

Source of Support: Nil

Competing Interests None

\section{CLASSICAL LICHEN PLANUS AND LICHEN PLANUS PIGMENTOSUS INVERSUS OVERLAP WITH DERMOSCOPIC FEATURES}

\author{
Şule Güngör ${ }^{1}$, İlteriş Oğuz Topal ${ }^{1}$, Şenay Erdoğan ${ }^{2}$, Deniz Özcan ${ }^{2}$

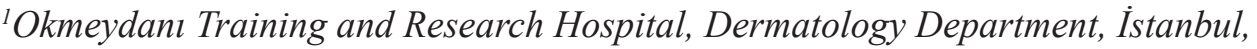 \\ Turkey \\ ${ }^{2}$ Okmeydanı Training and Research Hospital, Pathology Department, İstanbul, Turkey \\ Corresponding author: Dr Şule Güngör \\ drsulegungor@yahoo.com
}

\begin{abstract}
A 35-year-old man was referred to our hospital with a 3 months history of itchy cutaneous eruption on the trunk and asymptomatic cutaneous eruption on both groins. Physical examination revealed several, purplish-brown, scaly papules on the trunk and well-circumscribed, brown patchs in a linear distribution, on the bilateral inguinal regions. Dermoscopic examination of papules on the trunk revealed white crossing lines surrounded by brown dots; dermoscopic examination of patchs on groins revealed gray-brown dots and globules. According to histological, dermoscopical and clinical changes, the diagnoses of classical lichen planus (LP) for the lesions on the trunk; and lichen planus pigmentosus inversus (LPPI) for the lesions on the inguinal regions were made. Inhere we have described a rare case of LPPI and classical LP with dermoscopic features; and we suggest that LPPI is a variant of classical LP.
\end{abstract}

Key words: Lichen planus; lichen planus pigmentosus inversus; dermoscopy

Cite this article:

Şule Güngör, İlteriş Oğuz Topal, Şenay Erdoğan, Deniz Özcan. Classical lichen planus and lichen planus pigmentosus inversus overlap with dermoscopic features. Our Dermatol Online. 2014; 5(1): 42-44.

\section{Introduction}

Lichen planus pigmentosus inversus (LPPI) presents with brownish macules and patches in flexural regions and known as a rare variant of lichen planus pigmentosus (LPP) [1]. LPP is an uncommon variant of chronic lichen planus (LP) that is characterized by hyperpigmented, dark-brown macules in sunexposed areas especially in Indian patients [2]. In here, we present a case of classical LP and LPPI overlap in a man, with dermoscopic features.

\section{Case Report}

A 35-year-old man was referred to our hospital with a 3 months history of itchy cutaneous eruption on the trunk and asymptomatic cutaneous eruption on both groins. He had no significant medical history. Physical examination revealed several, purplish-brown, scaly papules on the trunk (Fig. 1) and well-circumscribed, brown patchs in a linear distribution, on the bilateral inguinal regions (Fig. 2). Dermoscopic examination of papules on the trunk revealed white crossing lines surrounded by brown dots (Fig. 3); dermoscopic examination of patchs on groins revealed gray-brown dots and globules (Fig. 4). Oral, nail, scalp or genital lesions were absent. A causal relationship with drugs, recent sun exposure, or trauma could not be identified. Laboratory evaluation, including blood cell count, fasting blood sugar levels, liver function, serum electrolyte levels, serum electrophoresis, urinalysis were within normal limits. Biopsy specimen of the papul on the trunk showed hyperkeratosis, hypergranulosis, bandlike inflammatory lymphocytic infiltrate with basal vacuolar changes and melaninin continence in the upper dermis (Fig. 5), the second biopsy specimen of the macula on the inguinal region showed regressive pattern of lichen planus with prominent melaninin continence (Fig. 6). Histopathological diagnosis of the first biopsy was consistent with classical lichen planus, second biopsy was consistent with melanosis. According to histological, dermoscopical and clinical changes, the diagnoses of classical LP for the lesions on the trunk; and LPPI for the lesions on the inguinal regions were made. Betamethasone dipropionate ointment was applied once daily for 2 weeks. Classical LP lesions were resolved, but LPPI lesions revealed no change after 2 weeks. Tacrolimus ointment was applied twice daily for 4 weeks on the inguinal region and no change was observed in LPPI lesions. 


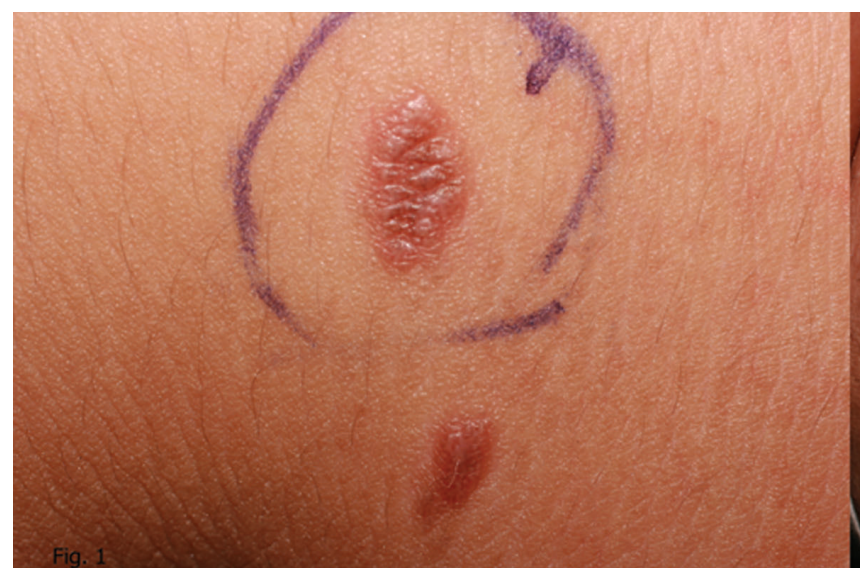

Figure 1. Purplish-brown, scaly papula on the trunk.

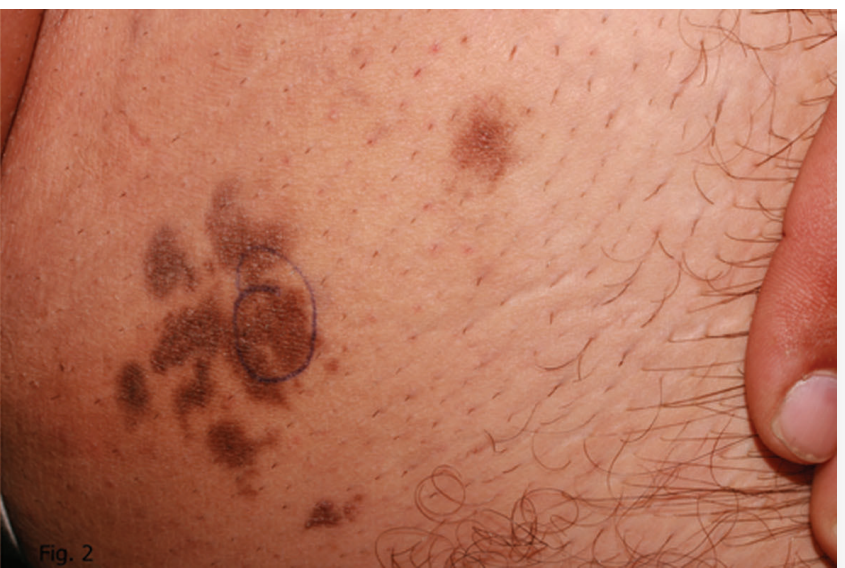

Figure 2. Well-circumscribed, brown patchs in a linear distribution, on the inguinal region.

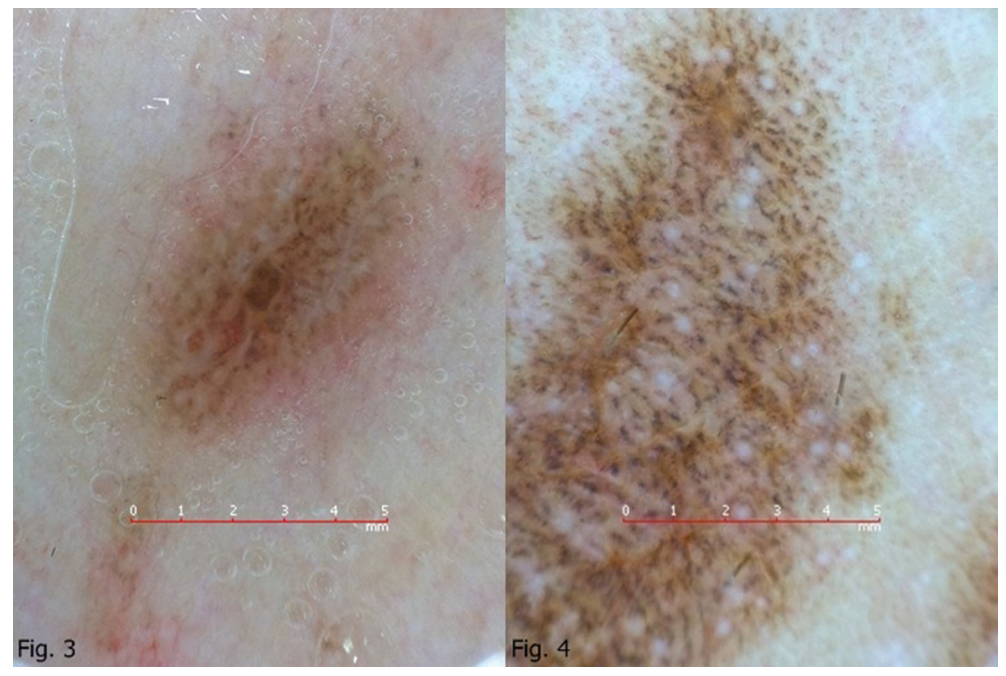

Figure 3. White crossing lines surrounded by brown dots on dermoscopic examination.

Figure 4. Dermoscopic examination of patchs on groins revealed gray-brown dots and globules.

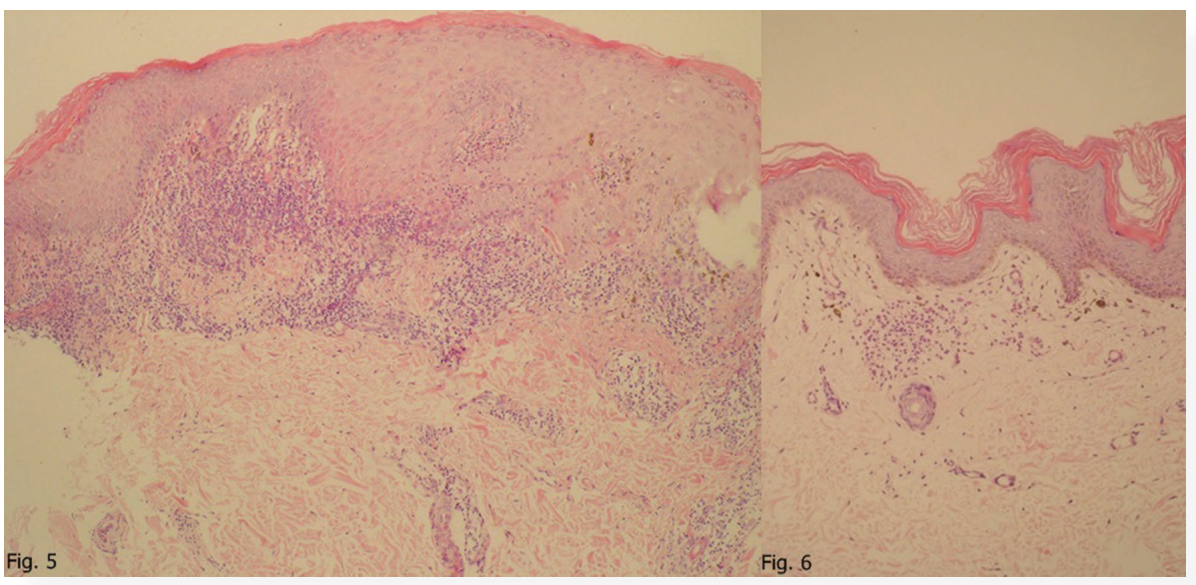

Figure 5. Biopsy specimen of the papul on the trunk showed hyperkeratosis, hypergranulosis, bandlike inflammatory lymphocytic infiltrate with basal vacuolar changes and melaninin continence in the upper dermis.

Figure 6. Biopsy specimen of the macula on the inguinal region showed regressive pattern of lichen planus with prominent melaninin continence. 


\section{Discussion}

The eruption of LPPI occurs mainly in the flexural regions and presents with brownish macules and patches. It is known as a rare variant of LPP [1].

LPP is an uncommon variant of chronic lichen planus that is characterized by hyperpigmented, dark-brown macules in sunexposed areas especially in Indian patients [2].

The first literature of classic LP and LPPI coexistence was reported by Saray et al. [3]. Their patient's LPPI lesions were resistant to oral steroid treatment, but classical LP lesions resolved. Similar to that case, our patient's LPPI lesions were resistant to both therapies, while classic LP lesions were resolved by treatment. In our case, dermoscopic examination of the papules on the trunk showed white crossing lines corresponding to the Wickham striae, histopathological examination was consistent with classic LP and resolved by corticosteroid ointment. On the other hand the macules on the groins showed gray-blue dots and globules corresponding with dermal melanophages, and histopathological examination was consistent with LPPI and resistant to treatment. Though our patient states that all lesions (both on the trunk and groins) appeared around the same time, they were clinically and histopathologically different. We consider that it depends on the localization of the lesions, that the course of the lesions on the intertriginous areas alters by friction; showing less lichenoid reaction, more pigment incontinence resulting in asymptomatic brown macules and patches, instead of pruritic purplish papules in classic LP. Due to less inflammation, LPPI lesions are resistant to anti-inflammatory drugs.
Other three cases of LLPI - classical LP relation were reported by Kim and al [4,5]. Their patient's lesions were reported to have emerged on the long standing LP inversus.

Current case is the fifth case in which classic LP and LPPI overlap. In this situation, we doubt whether LPPI is a variant of LPP or it originates from classical LP. Considering this claim, it seems that LPPI is not a variant of LPP, it is rather a variant of classical LP; but the course of the lesions alters by friction. In conclusion, we have described a rare case of LPPI and classical LP with dermoscopic features; and we suggest that LPPI is a variant of classical LP instead of LPP.

\section{REFERENCES}

1. Pock L, Jelínková L, Drlík L, Abrhámová S, Vojtechovská S, Sezemská D, et al. Lichen planus pigmentosus-inversus. J Eur Acad Dermatol Venereol. 2001;15:452-4.

2. Kanwar AJ, Dogra S, Handa S, Parsad D, Radotra BD. A study of 124 Indian patients with lichen planus pigmentosus. Clin Exp Dermatol. 2003;28:481-5.

3. Saray Y, Güleç T, Seçkin D. Lichen planus pigmentosus: report of four cases. T Klin J Dermatol. 2004;14:222-6.

4. Kim BS, Aum JA, Kim HS, Kim SJ, Kim MB, Oh CK, et al. Coexistence of classic lichen planus and lichen planus pigmentosusinversus: resistant to both tacrolimus and clobetasol propionate ointments. J Eur Acad Dermatol Venereol. 2008;22:106-7.

5. Kim BS, Park KD, Chi SG, Ko HC, Lee WJ, Lee SJ, et al. Two cases of lichen planus pigmentosus-inversus arising from longstanding lichen planus-inversus. Ann Dermatol. 2008;20:254-6.

Copyright by Şule Güngör, et al. This is an open access article distributed under the terms of the Creative Commons Attribution License, which permits unrestricted use, distribution, and reproduction in any medium, provided the original author and source are credited. 